\title{
MÉTRICA Y RETÓRICA (JUEGO DE PALABRAS) EN LA POESÍA ESPAÑOLA CONTEMPORÁNEA
}

\begin{abstract}
Resumen. El presente estudio Métrica y retórica (juego de palabras) en la poesía española contemporánea es un intento de conciliar la métrica (ritmo, rima, etc.) y la retórica. Para ello, se selecciona un conjunto de figuras retóricas que cumplen efectos rítmicos en el discurso poético versificado: el calambur, la paronomasia, el eco y la aliteración. El último capítulo se dedica al fenómeno fónico-rítmico del encabalgamiento cuando se emplea con una finalidad lúdica, por lo que funciona como un atípico juego de palabras. El corpus de ejemplos está compuesto por textos de la poesía española contemporánea.
\end{abstract}

Palabras clave: juego de palabras, métrica, figura retórica, lírica española.

\section{Introducción}

Aunque hay numerosos estudios sobre los juegos de palabras en la literatura (también en otras clases de discursos, como el periodismo y la publicidad), no se ha prestado atención suficiente a la intervención de estos en la consecución de las categorías rítmicas de los textos versificados: figuras como la paronomasia, el eco o el políptoton sustentan muchas veces el ritmo diseñado por el poeta en la composición, en especial el ritmo de timbres (rima) y el de cantidad (metro). No obstante esta desatención, no puede obviarse que están ampliamente reconocidas por la tradición literaria ciertas convenciones métricas relacionadas con el juego verbal, como, por ejemplo, entre las rimas intensas (Baehr, 1970), la rima homónima y la rima parónima, tildadas a veces de toscas o fáciles, que se basan, como es sabido, en la antanaclasis y la paronomasia, respectivamente (adviértase, no obstante, que la rima homónima ha de ser consonante, pero no necesariamente la parónima: puede ser asonante

\footnotetext{
* Universidad Nacional de Educación a Distancia.
} 
o casi consonante según la posición de los sonidos recurrentes y los no recurrentes). Asimismo, forman parte del código estilístico tradicional otras figuras fónicas y licencias métricas, como el homoiotéleuton o similiter desinens y el homeóptoton o similiter cadens (recursos englobados a veces en el fenómeno conocido como similicadencia, como término genérico), o la diéresis, la sinéresis, etc. (Mayoral, 1994; Domínguez Caparrós, 2014, etc.).

Las figuras retóricas que intervienen en la rima son fundamentalmente de naturaleza fónica, $y$, más raramente, morfológica y léxica (derivatio, falsa etimología, homeóptoton, políptoton, rima idéntica, etc.), si bien puede celebrarse el concurso de mecanismos de otra naturaleza, como el paralelismo, el hipérbaton, el quiasmo, la antimetábole o el retruécano (en el sentido adoptado en Lázaro Carreter, 1971, entre otros autores), de orden sintáctico, por cuanto que la distribución de los constituyentes sintácticos convenida por el poeta puede estar al servicio de la correspondencia rimante. Asimismo, otras figuras fónicas de repetición, como la aliteración (en cualquiera de sus variantes, como el parómeon u homopróforon, la geminada, etc.), que no representan en sí mismas, de modo regular, un juego de palabras, pueden promover o potenciar alguna clase de ritmo, incluida la rima. Ello se pone de manifiesto, por ejemplo, cuando se resalta la figura aliteración, bien con la acumulación o multiplicación de segmentos aliterativos, bien con la conjugación de varias series aliterativas, a veces dispuestas deliberadamente en el verso de una forma ordenada o dentro de las palabras aliteradas, bien, simplemente, cuando la aliteración es intensa (la repetición no es de un único sonido, sino de dos, de tres o de más; rayana, entonces, en la paronomasia). Igualmente, el encabalgamiento, elemento generador de ritmo y, al mismo tiempo, fenómeno regularmente insensible al efecto lúdico, puede habilitarse para promover un juego verbal (como la ruptura de una frase hecha o de alguna suerte de isotopía), justo en el lugar del verso donde se materializa habitualmente la rima en la versificación regular.

En este trabajo nos proponemos mostrar la comunión de las categorías métricas y las figuras retóricas capaces de generar un juego verbal. Para ello, seleccionaremos un corpus representativo de textos que contengan algún mecanismo de esta suerte. El corpus que presentamos es solo un botón de muestra de los centenares de textos que hemos recogido-que, obviamente, no serán todos los existentes-. Los textos no pertenecen solo a los poetas más proclives al juego verbal, como Vicente Huidobro, Rafael Alberti, Carlos Edmundo de Ory, Gloria Fuertes, Blas de Otero..., sino a una diversidad más amplia: Vicente Aleixandre, Jorge Guillén, Miguel Hernández, José Hierro, Félix Grande, Emilio Prados, Federico García Lorca, Luis Cernuda, Antonio Machado, Manuel Machado, Miguel de 
Unamuno, Eugenio García de Nora, Ramón de Garciasol, Luis Rosales, Leopoldo María Panero, Carlos Murciano, Carlos Bousoño, Claudio Rodríguez... Limitamos la muestra a la poesía española contemporánea (siglo XX), incluyendo la literatura hispanoamericana, de la que hemos registrado algunos autores, como Pablo Neruda, Octavio Paz y César Vallejo.

Las «figuras de estilo» que hemos elegido para ilustrar la conjunción entre métrica y retórica son el calambur, la paronomasia, el eco y la aliteración; todas pueden adscribirse al grupo de figuras fónicas de repetición relajada, conforme a la terminología de Lausberg (1966-1967). Además, incluimos el fenómeno rítmico del encabalgamiento cuando ejemplifica esa comunión entre métrica y retórica, la métrica al servicio del juego verbal.

\section{La relación entre la métrica y la retórica}

\subsection{Calambur}

Para la ilustración de esta figura, adoptamos el sentido de calambur propuesto inicialmente en Lázaro Carreter (1971), reformulado y enriquecido posteriormente en García-Page (1990a, 2010), y seguido por otros autores (Mayoral, 1994). No se sigue, pues, la concepción del término como sinónimo de juego de palabras, equívoco, retruécano 'juego de palabras' o pun que adoptan otros autores; especialmente, en la bibliografía francesa e inglesa (cfr., por ejemplo, Serra, 2000: 386-387). Como también es sabido, el tipo de procedimiento formal que sustenta nuestro calambur se analiza también a veces como disociación (Llano, 1984) o, en el ámbito francés, mot-gigogne (Dubois y otros, 1982), en especial textos como el que ilustra el ejemplo 5).

La repetición que da fundamento a este fenómeno no es tan «relajada» como la que configura la paronomasia o la aliteración, por ejemplo; es más, la rima que representa podría analizarse como homónima por la aparente identidad fónica entre las series homófonas conformantes del juego (la diferencia fónica reside esencialmente en la juntura entre los constituyentes gráficamente separados que presenta una de las secuencias homófonas): 
(1) Los poemas (¿son poemas?) no tienen orden ni concierto, -sé que a veces desconciertopero están escritos con cierto amor (Gloria Fuertes)

(2) Difícil, por ahora, ser demente porque yo no escribo de mente (Gloria Fuertes)

(3) Agota.

Agota.

¡iEste gota a gota!! (Gloria Fuertes)

(4) Con las flores de mi ramo

puesto en agua

el crujido de la enagua

y el chasquido de los besos (Manuel Machado)

(5) Y en el juego angustioso de un espejo frente a otro cae mi voz

y mi voz que madura

y mi voz quemadura

y mi bosque madura

y mi voz quema dura

como el hielo de vidrio

como el grito de hielo (Xavier Villaurrutia)

En otros textos, los escritores sitúan el juego verbal en otros lugares rítmicamente destacados del poema versificado distintos del final de verso donde se manifiesta principalmente la rima: en 6) y 7), configurando una epanalepsis o epanadiplosis; en 8), una anadiplosis o cadena:

(6) el hecho de haber sido helecho

no condiciona al hecho (Gloria Fuertes)

(7) Adiós hay que decir a Dios (Vicente Huidobro)

(8) ... Ondulan silvestres: «Mira: flores».

Miraflores. La reina bautizó los cubiles (Vicente Aleixandre)

\subsection{Paronomasia}

La paronomasia es, sin ningún género de dudas, la figura fónica más prolífica, el mecanismo de juego más socorrido para representar el ritmo de timbres. 
Con frecuencia apoyada en el paralelismo sintáctico y métrico, e incluso en la repetición léxica, que favorecen notablemente el resalte del efecto estilístico, puede aparecer al final de los dos miembros o (supuestos) hemistiquios de un verso compuesto o bimembre, sea de arte mayor o menor, es decir, al medio y al final de verso, cerrando sartas simétricas $(9-13,23)$, solo al comienzo de verso, como una anáfora imperfecta (14-15, $24)$, o solo al final de verso, como una epífora imperfecta $(16-18,22)$. Como un juego de artificio más complejo, los poetas utilizan a veces dos series paralelas, de manera sucesiva o simultánea, de paronomasias: por ejemplo, en el centro (19), en el centro y al final (20-21), en lugares diversos (26). No menos artificioso es el aprovechamiento completo de una sola serie o figura paronomásica, como ilustran los textos de Felipe Boso (22), Gabino Alejandro Carriedo (23) y Gloria Fuertes (24), mediante la conmutación sucesiva del primer sonido vocálico de una pieza léxica (apofonía); este juego acrobático podría describirse como una variedad de la cadena. Otra clase de cadena es la que conforma la serie de parónimos rimados del texto 25); los tres constituyentes están en relación de inclusión sucesiva: oro $>$ coro $>$ decoro. Se reviste también de un alto índice de artificiosidad la figura paronomásica que se obtiene mediante la inversión de los sonidos conformantes (26-28), paronomasia que se viene describiendo comúnmente como anagrama (adviértase que en [26], similarmente a la que presentan los textos [19-21], se construyen dos series sucesivas de paronomasias; la segunda constituye, a su vez, otro juego verbal, pues se erige en un mecanismo de ruptura de una expresión fija, la locución adverbial poco a poco); el texto de Cirlot representa la explotación máxima del recurso, un mero juego circense con el significante fónico-gráfico: VieNe / NieVe, PoCo / CoPo, hEmbr A / hAmbrE, Inger / Ingre / Inegr / Inreg / Inrge... La acumulación o apilamiento de paronomasias (textos 29-31) representa un derroche pirotécnico del artificio: la paronomasia de 29) ilustra la figura conocida como falsa etimología; 30) es un ejemplo de aglomeración de paronomasias organizadas en series distintas que se van sucediendo verso a verso; 31) ilustra una de las manifestaciones de la rima en eco, solo que los componentes que configuran el eco son parónimos (cama y ama, en este orden, forman un eco perfecto, no así cita y resucita: el eco se lograría invirtiendo el orden de sucesión de los parónimos):

(9) Canten tus alas, canten tus olas (Rafael Alberti)

(10) que también aspirase, que también esperase (Vicente Aleixandre)

(11) para cantar al viento, para cantar al verso (Dámaso Alonso) 
(12) la cuña de los años, la coña de los cantos (Gabriel Celaya)

(13) Suena la soledad de Dios. Sentimos la soledad de dos (Blas de Otero)

(14) Sal de mi tierra, sol de mi cielo (Miguel de Unamuno)

(15) cúmulo de sierpes túmulo de lava (Gloria Fuertes)

(16) otros hombres, otros nombres (Ramón de Garciasol)

(17) Pierdes la chaveta cambias la chaqueta (Gloria Fuertes)

(18) de mágicos lagos en tristes jardines y enfermos jazmines (Antonio Machado)

(19) Que en los penales no haya ningún justo Que en los panales no haya ningún zángano Que en las trazas no haya hipocresía Que en los trece no haya desengaño (Gloria Fuertes)

(20) para todas las cintas más distantes para todas las citas más distintas (César Vallejo)

(21) Con el alma al hombro

bajo el olmo, el hombre (Gloria Fuertes)

(22) Los años pasan, Los años pesan, Los años pisan, Los años posan, ¿Por qué no pusan los años? (Felipe Boso)

(23) Daba pasos, daba pesos, pisos daba, daba posos (G. Alejandro Carriedo)

(24) Yo, remera de barcas, ramera de hombres, romera de almas, rimera de versos (Gloria Fuertes)

(25) Muchas veces se está solo pero mejor con decoro ¡A la mierda el oro y a la mierda el coro! (Gloria Fuertes) 
(26) Viene la nieve.

Cae

poco

a

copo (Blas de Otero)

(27) Y me avergüenzo de un libro lujoso de poemas encuadernado en piel de hembra

ante tanta hambre (Gloria Fuertes)

(28) Inger

Ingre

Inegr

Inreg

Inrge

[...] (Juan-Eduardo Cirlot)

(29) Si canto soy un cantueso

Si leo soy un león

Si emano soy una mano

Si amo soy un amasijo

Si lucho soy un serrucho (Carlos Edmundo de Ory)

(30) Blusa de Blasa.

Solo en el polo.

Garra de la guerra.

Qué asco de casco.

Cuento que encanta.

Pato a la puta.

Pena de pene.

Pancha y su Pancho.

Vista a la bestia.

La tía teta.

Los senos sanos.

Siete de sota.

Leche en el lecho.

Nicho en la noche (Gloria Fuertes)

(31) Si de algo positivo es la certeza: cerveza.

Si de algo negativo incertidumbre: lumbre.

... La improbabilidad es mucha: lucha.

Aunque tu amor no te dé cama: ama.

Si tu amor no quiere verte: Muerte.

$\mathrm{Si}$ ave nueva te da cita: resucita. (Gloria Fuertes) 


\subsection{Eco}

La figura eco es precisamente el juego verbal practicado en textos como los que se citan a continuación, donde acaso no tenga efecto sobre ninguna manifestación del ritmo, o su efecto sea apenas perceptible (Mayoral, 1994: 72-73; García-Page, 2003: 51-56, 2009, 2013a: 29-31, 2013b). Aquí prestamos atención a una de las posibles manifestaciones del eco: una palabra, la palabra-eco, reproduce fielmente el segmento final de la palabra precedente (cfr. 31):

(32) donde su dentadura dura muerde (Miguel Hernández)

(33) Aquí yace Altazor azor fulminado por la altura (Vicente Huidobro)

(34) Segundo esputo puto de tu progenitora (Rafael Alberti)

En cambio, sí puede influir en el ritmo o la métrica en los siguientes casos, donde el eco es resaltado a veces por distribuir los constituyentes sucesivos (en realidad, parónimos) al final de un verso y al comienzo del siguiente, esto es, configurando una anadiplosis y, en algunos casos, con encabalgamiento (35-41).

Que se trata de un recurso deliberadamente empleado, diseñado previamente por el poeta, lo prueba, además de la selección del léxico y de la ubicación (lugares métricamente privilegiados), la propia iteración recurrente del elemento que crea el eco (41), incluso del mismo sustantivo eco (40).

(35) ¡Ay mísera de ti! jay española ola lejana! Sálvame contigo (Blas de Otero)

(36) Cuando voy por la calle o bien en algún pueblo con palomas, lomas y puente romano (Blas de Otero)

(37) Odio el ágora la odio odio también la palestra odio el musgo el licopodio odio la mano maestra (Carlos Edmundo de Ory)

(38) ¡Elevadme, embebedme! ¡Vedme, resucitadme! (Carlos Bousoño)

(39) Esgrime tu crespada espada (Miguel Hernández) 
(40) Ínclitas guerras paupérrimas, sangre infecunda perdida. (No sé nada, nada). Ganada (no sé) nada, nada: éste es el seco eco de la sangre (Blas de Otero)

(41) Pespunte de seda virgen tu canción.

Abejaruco.

Uсо исо исо исо (Federico García Lorca)

\subsection{Aliteración}

El tipo de repetición que determina la figura llamada aliteración no es siempre el mismo; dicho de otra manera, el término aliteración se ha interpretado diversamente; no existe una definición única. En algunas concepciones (por ejemplo, en ciertos autores del ámbito anglosajón), se limita a la iteración de los sonidos iniciales de dos o más palabras, fenómeno que, en la tradición retórica, se conoce como parómeon u homopróforon (García-Page, 2009, 2013a: 31-34, 2013b, 2018). Por otra parte, la repetición de los sonidos finales, si bien puede interpretarse como aliteración, en realidad se describe comúnmente como rima imperfecta o similicadencia; son pocos los autores que la analizarían propiamente como aliteración. En un sentido más amplio, la aliteración sería la repetición de uno o varios sonidos en cualquier lugar de la palabra.

El parómeon se erige en artificio cuando el segmento fónico iterativo no es un sonido, sino dos o más, que a veces forman sílaba, como ilustran los versos 42)-44), hasta el punto de asemejarse a la paronomasia; la voluntad de juego por parte del poeta, de crear un efecto lúdico, es evidente en estos casos. La aliteración puede afectar al ritmo acentual:

(42) Otro año más. España en sombra. Espesa (Blas de Otero)

(43) Espaciosa y triste España (Blas de Otero)

(44) La pondré como una espada en un espejo (Pablo Neruda)

En los textos siguientes (45-46), la aliteración constituye un auténtico juego verbal: una única clase de aliteración -simple o múltiple (l-ol-al$b o l)$, exacta o con variante (ol-al, bol)- se extiende a lo largo de todo el poema [45] (adviértase, además, la aliteración de vibrantes); varias series aliterativas ( $p u$ / lla / ña / vi) se diseminan ordenadamente, escanciadas en versos consecutivos [46]. Hay un especial empeño del poeta en producir 
este juego seleccionando el léxico oportuno que contiene los sonidos recurrentes. La repetición múltiple crea un discurso poético lleno de sonoridad:

(45) Árboles abolidos, volveréis a brillar

al sol. Olmos sonoros, altos

álamos, lentas encinas,

olivo

en paz,

árboles de una patria árida y triste (Blas de Otero)

(46) Aragón, cúpula pura, danos

la paz.

Morella, uña mellada.

Peñafiel. Fuensaldaña.

Esla. Guadalquivir. Viva Sevilla (Blas de Otero)

Acaso la máxima expresión lúdica de la figura aliteración es la que ilustran los textos 47) y 48), meros malabarismos con el material fónico: el primero es una suerte de trabalenguas, sustentado básicamente en la iteración machacona de la consonante linguovelar sorda; el segundo representa un tautograma, conseguido mediante la multiplicación de la aliteración (parómeon) de la consonante nasal bilabial:

(47) Trajo fríjoles el hijo, rijas trajo, trajo tojos, trajo, trajo, trajo, trajo un trajín como un repollo.

Ay, qué hijo más canijo,

Ay, qué pijo más rijoso! (Gabino Alejandro Carriedo)

(48) Manolo mío:

Mi madrileño marchoso, maduro melocotón maleable macedonia mascaré mañana, mortadela moscatel mío.

Madrugaré maestro

-me manipulas-,

Manolo, macho mío,

mándote majuelas, magnolias

maíz, mijo,

-me matas, majo-. (Gloria Fuertes) 


\subsection{Encabalgamiento}

El fenómeno rítmico del encabalgamiento se reviste de carácter lúdico en determinados contextos, como en aquel caso en que un sintagma, una frase, una locución, un compuesto sintagmático... (algunos autores emplean el término de sirrema [Balbín, 1962]), es escindido en dos partes ubicadas en versos consecutivos y se da la circunstancia de que el segmento que cierra el verso encabalgado tiene sentido autónomo, como si el segmento desplazado al verso encabalgante fuera superfluo, y que dicho sentido queda cancelado, y reemplazado por otro, al proseguir la lectura del verso encabalgante. Podría decirse que el poeta forja o emplea una secuencia con dos sentidos o lecturas posibles según se escancie en uno o dos versos, según se segmente al final del primer verso, apoyándose en la pausa de cierre (por tanto, con encabalgamiento abrupto), o en un punto concreto del interior del segundo verso, renunciando a la pausa de cierre métrico (véase especialmente García-Page, 1991; asimismo, Bousoño, 1952: 484-485; Alarcos Llorach, 1967; Martínez García, 1975: 467-468; García-Page, 1990b: 240 ss., y 2003: 215-227). Es muy común que el segmento desplazado al segundo verso sea un adjetivo o sintagma preposicional con función de adyacente o complemento de un núcleo sustantivo, que queda colgando en el verso encabalgado, o un complemento del predicado (complemento directo, complemento circunstancial, etc.) si se trata de un sintagma verbal $(49,55,56,59,61$, etc.); puede producirse el fenómeno inverso, haciendo que el adjetivo o sintagma preposicional se interprete como elemento autónomo $(57,58$, 60 , etc.). Es lo que sucede en textos como los que se citan a continuación. El sentido primero que resulta de la lectura del verso con pausa métrica de cierre, sin encabalgamiento, viene favorecido normalmente por otra suerte de recursos, como la repetición, la enumeración, la simetría sintáctica, etc. $(49,52,55$, etc.), el tejido semántico del contexto o, en términos greimasianos, las isotopías $(53,54,57$, etc.), o meramente el carácter semánticamente autónomo de la frase, de la locución o de la palabra compuesta que se escancia en el primer verso (50, 51, etc.):

(49) Si las sílabas valen,

si la sibila acierta,

si los atletas pegan

patadas al balón (Gloria Fuertes)

(50) Después, como un cadáver puesto en pie

de guerra, clamaría por los campos (Blas de Otero) 
(51) Anda, levántate, España.

(Ponte

en pie

de guerra) (Blas de Otero)

(52) Árboles abolidos, volveréis a brillar

al sol. Olmos sonoros, altos

álamos, lentas encinas,

olivo (Blas de Otero)

(53) ¿Recuerdas? Te mondaba una manzana

y me corté en el dedo

del corazón; y tú, viendo la grana,

sentiste al pronto miedo (Miguel de Unamuno)

(54) En este Café

se sentaba don Antonio

Machado

silencioso

y misterioso, se incorporó

al pueblo,

blandió la pluma (Blas de Otero)

(55) ... Y como soy un pobre obrero

de la palabra, un mínimo minero

de la paz, no sé nada de la guerra (Blas de Otero)

(56) Prefiero

quedarme desnuda

de amigo insincero (Gloria Fuertes)

(57) ... cuando toca

mayo el campo de verde y rumores!

El compañero niño campesino,

tú, Juan, Francisco, Pedro, ¿con el vino

turbio de los fusiles? Labradores

indices te señalan. ¿Y la escuela

adonde fuimos juntos? (Ramón de Garciasol)

(58) Las palabras que velan el secreto

placer, y el labio virgen no lo sabe (Luis Cernuda)

(59) Una tarde imprevista, en el taller de un artista

vi a Mimí, débil y rubia, desnuda bajo la lluvia

de su cabello de oro

que era todo su tesoro (Manuel Machado) 
(60) Aquí vive un cura loco por un lindo adolescente (Antonio Machado)

(61) Y volver a nacer. Y arar la tierra del amor... (Blas de Otero)

(62) una juventud que vuela, una mujer y una casa, álamos en la ribera de la sangre, enredadera (Ramón de Garciasol)

A modo de ilustración, la doble lectura que provoca el juego verbal podría representarse, de manera gráfica, como sigue:

(49) pegan $\neq$ pegan patadas al balón

(50) puesto en pie $\neq$ (puesto) en pie de guerra

(51) ponte en pie $\neq$ (ponte) en pie de guerra

(52) Olmos sonoros, altos $\neq$ (Olmos sonoros, ) altos álamos

(53) (me corté en) el dedo $\neq$ (me corté en) el dedo del corazón

(54) don Antonio $\neq$ (don) Antonio Machado

(54) (En este Café se sentaba don Antonio Machado silencioso y misterioso,) se incorporó $\neq$ [...] se incorporó al pueblo

(55) (soy un pobre) obrero $\neq$ (soy un pobre) obrero de la palabra

(55) (soy un mínimo) minero $\neq$ (soy un mínimo) minero de la paz

(56) (quedarme) desnuda $\neq$ (quedarme) desnuda de amigo insincero

(57) labradores $\neq$ labradores indices

(58) (velan) el secreto $\neq$ (velan) el secreto placer

(59) (vi a Mimí, débil y rubia, desnuda bajo) la lluvia $\neq$ (vi a Mimí, débil y rubia, desnuda bajo) la lluvia de su cabello de oro

(60) un cura loco $\neq$ (un cura) loco por un lindo adolescente

(61) (arar) la tierra $\neq($ arar) la tierra del amor

(62) (álamos de) la ribera $\neq$ (álamos de) la ribera de la sangre 


\section{Conclusiones}

Nuestro estudio, ilustrado con 62 textos (de varios centenares registrados), demuestra que los poetas utilizan regularmente figuras retóricas generadoras de efectos lúdicos como constituyentes del ritmo de sus creaciones líricas; es el caso de la paronomasia, el eco, el calambur y la aliteración. También prueba que algunas categorías propias del discurso versificado, como el encabalgamiento, pueden producir dichos efectos. Por ello, nuestro estudio constituye un ejemplo de la conjunción de la retórica y la métrica.

Aunque el corpus de textos seleccionados pertenece a la poesía española contemporánea, la relación entre la métrica y la retórica en el sentido indicado es una constante en la literatura española desde sus comienzos. Son abundantes los testimonios en la poesía de los Siglos de Oro (Góngora, Quevedo, Cervantes, Lope de Vega, Salinas, Jáuregui, Medrano, etc.).

\section{Bibliografía}

ALARCOS LLORACH, E. (1967). «Secuencia sintáctica y secuencia rítmica», en E. ALARCOS LLORACH y otros (eds.), Elementos formales en la lírica actual. Santander: UIMP, 9-16.

BAEHR, R. (1970). Manual de versificación española. Madrid: Gredos.

BALBÍN DE, R. (1962). Sistema de rítmica castellana. Madrid: Gredos, ${ }^{3} 1975$.

BOUSOÑO, C. (1952). Teoría de la expresión poética, 2 vols. Madrid: Gredos, ${ }^{2} 1976$. DOMÍNGUEZ CAPARRÓS, J. (2014). Métrica española. Madrid: UNED.

DUBOIS, PH. y otros (1982). La rhétorique des jeux des mots. Urbino: Università di Urbino. Centro Internazionale di Semiotica et di Lingüística. Documents de travail et prè-publications 116-117-118, serie $B$.

GARCÍA-PAGE, M. (1990a). «Algunas observaciones acerca del calambur», en Investigaciones semióticas III. Madrid: UNED, I, 431-448.

GARCÍA-PAGE, M. (1990b). «Juegos lingüísticos en Gloria Fuertes (poesía)», Rilce, 6: 2, 211-243.

GARCÍA-PAGE, M. (1992). «Datos para una tipología de la paronomasia», Epos. Revista de filología, 8, 155-243.

GARCÍA-PAGE, M. (2003). El juego de palabras en la poesía de Gloria Fuertes. Madrid: UNED.

GARCÍA-PAGE, M. (2004). «Juegos idiomáticos en la poesía contemporánea», en R. SENABRE y otros (eds.), El lenguaje de la literatura (siglos XIX y XX). Salamanca: Ambos Mundos, 113-176. 
GARCÍA-PAGE, M. (2009). «Blas de Otero: la 'poesía sonora'», en M. GARCÍAPAGE (ed.), Poesía española contemporánea (siglo XX). Ocho poetas, ocho estudios de lengua literaria. Lugo: Axac, 101-144.

GARCÍA-PAGE, M. (2010). «El calambur: una propuesta de definición», Moenia. Revista lucense de lingüistica \& literatura, 16, 167-194.

GARCÍA-PAGE, M. (2013a). «Juegos verbales en la literatura contemporánea», Colindancias. Journal of the Network of Hispanists from Hungary, Romania and Serbia, 4, 9-40.

GARCÍA-PAGE, M. (2013b). «De los sonidos de la poesía: aliteración y eco en Blas de Otero», en M. A. PENAS IBÁÑEZ (ed.), Panorama de la fonética española actual. Madrid: Arco / Libros, 487-506.

GARCÍA-PAGE, M. (2018). «El parómeon. Un apunte al Diccionario español de términos literarios internacionales (Garrido Gallardo, 2009)», en L. ALBURQUERQUE GARCÍA y otros (eds.), Vir bonus dicendi peritus. Homenaje al profesor Miguel Ángel Garrido Gallardo. Nuevos comentarios de textos hispánicos. Madrid: CSIC, 899-911.

LAUSBERG, H. (1966-1967). Manual de retórica literaria, 3 vols. Madrid: Gredos.

LÁZARO CARRETER, F. (1971). Diccionario de términos filológicos. Madrid: Gredos.

LLANO, M. T. (1984). La obra de Quevedo. Algunos recursos humorísticos. Salamanca: Universidad de Salamanca.

MARTÍNEZ GARCÍA, J. A. (1975). Propiedades del lenguaje poético. Oviedo: Universidad de Oviedo.

MAYORAL, J. A. (1994). Figuras retóricas. Madrid: Síntesis.

SERRA, M. (2000). Verbalia. Juegos de palabras y esfuerzos de ingenio literario. Barcelona: Atalaya. 